Visión Antataura

Vol.4, No.1: 52-65

Junio - Noviembre, 2020

Panamá

ISSN 2520-9892

\title{
El Teorema Fundamental del Cálculo en la Teoría de Integración de Riemann
}

\section{The Fundamental Theorem of Calculus in the Riemann Integration Theory}

\author{
Ángela Yaneth Franco ${ }^{1}$
}

${ }^{1}$ Maestría en Matemática Educativa; Profesora, Universidad de Panamá, Centro Regional Universitario de Veraguas y Universidad Tecnológica de Panamá, Centro Regional de Veraguas; angela06franco@hotmail.com

Resumen: El teorema fundamental del cálculo provee una formalización genérica de la relación inversa entre las teorías de diferenciación e integración; a saber,

$$
\frac{d}{d x}\left(\int_{a}^{x} f(t) d t\right)=f(x)
$$

y

$$
\int_{a}^{x} f^{\prime}(t) d t=f(x)-f(a)
$$

lo cual se satisface en la teoría de integración de Cauchy. Sin embargo, el teorema fundamental del cálculo pierde sentido si la derivada $f^{\prime}(x)$ de una función derivable no es Riemann integrable. El objetivo de este artículo es presentar las propiedades que debe satisfacer la derivada $f^{\prime}(x)$ de una función diferenciable para que se satisfaga el Teorema Fundamental del Cálculo en la teoría de integración de Riemann.

Palabras clave: Integral de Riemann, teorema fundamental del cálculo, diferenciabilidad, integrabilidad, primitiva.

Abstract: The fundamental theorem of calculus provides a generic formulation of the inverse relationship between the theories of differentiation and integration; meaning,

and

$$
\frac{d}{d x}\left(\int_{a}^{x} f(t) d t\right)=f(x)
$$

$$
\int_{a}^{x} f^{\prime}(t) d t=f(x)-f(a)
$$

which is satisfied in Cauchy integration theory. However, the fundamental theorem of calculus has no meaning if the derivative $f^{\prime}(x)$ of a differentiable function is not Riemann integrable. The purpose of the research is to preset the proprieties which should fulfill the derivative $f^{\prime}(x)$ of a differentiable function in order to satisfy the Fundamental Theorem of Calculus in the Riemann integration theory.

Key words: Riemann integral, fundamental theorem of calculus, differentiability, integrability, primitive. 


\section{Introducción}

Una de las principales ventajas de la teoría de integración de Cauchy (para funciones continuas) es que le permitió demostrar generalmente la existencia de integrales o funciones primitivas, cuyas propiedades, él pensó, podían ser estudiadas solo después de la demostración de existencia. Para funciones continuas $f:[a, b] \rightarrow \mathbb{R}$, Cauchy consideró la función $F:[a, b] \rightarrow \mathbb{R}$ definida por

$$
F(x)=\int_{a}^{x} f(t) d t
$$

Como por el Teorema del Valor Medio para Integrales, existe un $\theta \in[0,1]$ tal que

$$
\frac{F(x+h)-f(x)}{h}=\frac{1}{h} \int_{x}^{x+h} f(t) d t=f(\theta x+h)
$$

$F$ no es solo continua, sino también diferenciable en $[a, b]$. De aquí, Cauchy estableció los siguientes resultados (Barthle, 2011; Edwards, 2013; Bressoud, 2011).

Teorema 1: (Primera versión del Teorema Fundamental del Cálculo): $F$ es una función primitiva para $f$ en $[a, b]$; es decir, $F^{\prime}(x)=f(x)$ para todo $x \in[a, b]$.

Teorema 2: (Segunda versión del Teorema Fundamental del Cálculo): Todas las primitivas de $f$ deben ser de la forma $\int_{a}^{x} f(t)+C$, donde $C$ es una constante; esto es, si $G$ es una función con derivada continua $G^{\prime}$, tal que $G^{\prime}(x)=f(x)$, para todo $x \in[a, b]$, entonces

$$
\int_{a}^{x} f(t) d t=\int_{a}^{x} G^{\prime}(t) d t=G(x)-G(a)
$$

En la demostración del Teorema 2, Cauchy usa las hipótesis

a) $G$ tiene derivada en $[a, b]$

b) Esta derivada es continua en $[a, b]$

Para establecer que

$$
\int_{a}^{b} G^{\prime}(x) d x=G(b)-G(a)
$$

La pregunta a plantearse es:

¿Se pueden generalizar los teoremas 1 y 2 (debilitando las hipótesis) a la teoría de integración de Riemann? 
Como ilustración se presentan los siguientes ejemplos

Ejemplo 1: Sea $F:[-2,2] \rightarrow \mathbb{R}$ la función definida por

$$
F(x)= \begin{cases}x^{2} \operatorname{sen}\left(\frac{\pi}{x}\right) & , x \neq 0 \\ 0 \quad, x=0\end{cases}
$$

Note que $F$ es derivable en $[-2,2]$ y

$$
f(x):=F^{\prime}(x)= \begin{cases}2 x \operatorname{sen}\left(\frac{\pi}{x}\right)-\pi \cos \left(\frac{\pi}{x}\right), & x \neq 0 \\ 0 & , x=0\end{cases}
$$

Además $|f(x)| \leq 8$, para todo $x \in[-2,2]$.

Considere, la sucesión $\left\{\frac{1}{2 n}\right\}_{n=1}^{\infty}$ en $[-2,2]$. Entonces

$$
f\left(\frac{1}{2 n}\right)=2\left(\frac{1}{2 n}\right) \operatorname{sen}(2 n \pi)-\pi \cos (2 n \pi)=-\pi
$$

Como

$$
\lim _{n \rightarrow \infty}\left(\frac{1}{2 n}\right)=0 \quad \text { y } \quad \lim _{n \rightarrow \infty} f\left(\frac{1}{2 n}\right)=-\pi \neq f(0)
$$

se tiene que $f$ no es continua en $[-2,2]$. Sin embargo, $f$ es Riemann integrable en $[-2,2]$ y

$$
\begin{aligned}
\int_{-2}^{2} f(x) & d x=F(2)-F(-2) \\
& =4 \operatorname{sen}\left(\frac{\pi}{2}\right)-4 \operatorname{sen}\left(-\frac{\pi}{2}\right) \\
& =4+4 \\
& =8
\end{aligned}
$$

Así pues, la función $F$ satisface la segunda versión del Teorema Fundamental del Cálculo en $[-2,2]$, sin ser $F^{\prime}$ una función continua en $[-2,2]$.

Ejemplo 2: Sea $F:[-1,1] \rightarrow \mathbb{R}$ la función definida por

$$
F(x)= \begin{cases}x^{2} \operatorname{sen}\left(\frac{1}{x^{2}}\right) & , x \neq 0 \\ 0 & , x=0\end{cases}
$$

Note que $F$ es derivable en $[-1,1]$ y 


$$
f(x):=F^{\prime}(x)=\left\{\begin{array}{l}
2 x \operatorname{sen}\left(\frac{1}{x^{2}}\right)-\frac{2}{x} \cos \left(\frac{1}{x^{2}}\right), x \neq 0 \\
0, x=0
\end{array}\right.
$$

Considere la sucesión $\left\{\frac{1}{\sqrt{2 n \pi}}\right\}_{n=1}^{\infty}$ en $[-1,1]$. Entonces

$$
f\left(\frac{1}{\sqrt{2 n \pi}}\right)=\frac{2}{\sqrt{2 n \pi}} \operatorname{sen}(2 n \pi)-2 \sqrt{2 n \pi} \cos (2 n \pi)=-2 \sqrt{2 n \pi}
$$

Esto implica que la función $f$ no es Riemann integrable en $[-1,1]$, ya que no es acotada en $[-1,1]$. Por consiguiente, la función $F$ no satisface la segunda versión del Teorema Fundamental del Cálculo en $[-1,1]$.

De los dos ejemplos anteriores, se puede afirmar que:

i) La derivada de una función en un intervalo cerrado $[a, b]$ no es necesariamente Riemann integrable en $[a, b]$.

ii) La continuidad de la derivada $F^{\prime}$ de una función derivable $F$ no se puede tomar como un hecho.

iii) La hipótesis de continuidad de $F^{\prime}$ no es una condición necesaria para que se cumpla el Teorema 2.

La pregunta ahora es:

¿Qué condiciones debe satisfacer la derivada $F^{\prime}$ de la función $F$ que garantice la veracidad del Teorema 2?

Para contestar esta pregunta se estudiarán primero algunas propiedades que tiene la derivada $f^{\prime}$ de una función diferenciable $f$ definida en un intervalo $[a, b]$.

\section{La Propiedad del Valor Intermedio para Derivadas}

Sea $f:[a, b] \rightarrow \mathbb{R}$ una función diferenciable. De los Ejemplos 1 y 2 se sabe que $f^{\prime}$ no es necesariamente continua ni acotada en $[a, b]$. Así que la interrogante es, qué tan mal puede ser el comportamiento de la derivada de una función. En esta dirección el matemático francés Gastón Darboux (1847-1917) probó el siguiente teorema (Dunham, 2005; Propp, 2013). 
Teorema 3: Sea $f:[a, b] \rightarrow \mathbb{R}$ una función diferenciable en el intervalo $[a, b]$. Si $k$ es un número entre $f^{\prime}(a)$ y $f^{\prime}(b)$, entonces existe un $c \in[a, b]$ tal que $f^{\prime}(c)=k$.

\section{Demostración:}

Suponga que $f^{\prime}(a)<k<f^{\prime}(b)$. Considere la función $g:[a, b] \rightarrow \mathbb{R}$ definida por

$$
g(x)=k x-f(x)
$$

Como $f$ es diferenciable en $[a, b], g$ es diferenciable en $[a, b]$ y

$$
g^{\prime}(x)=k-f^{\prime}(x)
$$

Además, como $g$ es continua en $[a, b]$, existe un $c \in[a, b]$ tal que

$$
g(x) \leq g(c) \text {, para todo } x \in[a, b] .
$$

Por otro lado, como $g^{\prime}(a)=k-f(a)>0$ y

$$
g^{\prime}(a)=\lim _{h \rightarrow 0^{+}} \frac{g(a+h)-g(a)}{h}>0
$$

Se tiene que

$$
g(a+h)-g(a)>0, \text { cuando } h \rightarrow 0^{+}
$$

y

$$
g(a+h)>g(a), \text { cuando } h \rightarrow 0^{+}
$$

por lo tanto $a \neq c$.

De igual manera se tiene que $b \neq c$. Así pues, $c \in(a, b)$. Como $g$ alcanza su valor máximo en $c$ y $c \in(a, b)$, se tiene que $g^{\prime}(c)=0$. Es decir,

$$
k-f^{\prime}(c)=0 \quad \text { y } \quad k=f^{\prime}(c) .
$$

Ejemplo 3: Sea $f:[-3,3] \rightarrow \mathbb{R}$ la función definida por

$$
f(x)=\left\{\begin{array}{lr}
0, & -3 \leq x<0 \\
4, & 0 \leq x \leq 3
\end{array}\right.
$$

y considere la función $F:[-3,3] \rightarrow \mathbb{R}$ definida por

$$
F(x)=\int_{-3}^{x} f(t) d t
$$

Luego

$$
x<0 \Rightarrow F(x)=\int_{-3}^{x} f(t) d t=\int_{-3}^{x} 0 d t=0
$$




$$
\begin{aligned}
x=0 \Rightarrow F(0) & =\int_{-3}^{x} f(t) d t=\int_{-3}^{x} 0 d t=0 \\
x>0 \Rightarrow F(x) & =\int_{-3}^{x} f(t) d t=\int_{-3}^{0} f(t) d t+\int_{0}^{x} f(t) d t \\
= & 4 \int_{0}^{x} d t=4 x
\end{aligned}
$$

Así pues,

$$
F(x)=\left\{\begin{array}{l}
0,-3 \leq x<0 \\
4 x, \quad 0 \leq x \leq 3
\end{array}\right.
$$

Note que $F$ es continua, pero no diferenciable en $[-3,3]$, por lo que $F$ no es una primitiva de $f$ en $[-3,3]$. Más aun, por la Propiedad de Valor Intermedio para Derivadas (ver Teorema 3), $f$ no posee primitiva en $[-3,3]$, a pesar de que es Riemann integrable en $[-3,3]$. Recuerde que en el Ejemplo 2 se presentó una función que posee primitiva, pero no es integrable en el intervalo $[-2,2]$.

Los ejemplos anteriores fueron las bases que motivaron el replanteamiento del Teorema Fundamental del Cálculo en la teoría de integración de Riemann, lo cual se desarrolla en la siguiente sección.

\section{El Teorema Fundamental del Cálculo en la Teoría de Integración de Riemann.}

Para la función $f:[-1,1] \rightarrow \mathbb{R}$ definida en Ejemplo 2, se tiene que la derivada $F^{\prime}$ no es acotada en $[-1,1]$, por lo tanto, ella no es Riemann integrable en $[-1,1]$ y, por ende, no se satisface la segunda versión del Teorema Fundamental del Cálculo. Será que si se supone que $F^{\prime}$ es acotada en $[a, b]$, entonces se satisface el Teorema Fundamental del Cálculo. En este sentido se plantea la siguiente conjetura en la teoría de integración de Riemann. (Bressoud, 2011), (Botsko, 1991), (Propp, 2013).

Conjetura: Sea $F:[a, b] \rightarrow \mathbb{R}$ una función diferenciable en $[a, b]$ con derivada acotada $F^{\prime}$ en $[a, b]$. Entonces $F^{\prime}$ es Riemann integrable en $[a, b]$ y

$$
\int_{a}^{b} F^{\prime}(x) d x=F(b)-F(a)
$$


En 1881 el matemático italiano Vito Volterra (1860-1940) resolvió negativamente esta conjetura en su artículo "Sui principii del calcolo integrale ". (Edwards,2013), (Dunham,2005), (Brand, 1995). Ahí Volterra presentó un ejemplo de una función $F$ que tenía una derivada acotada en $[a, b]$ pero cuya derivada era tan discontinua como para ser Riemann integrable (o sea que el conjunto de discontinuidades tenía medida de Lebesque no nula); por lo tanto, la ecuación

$$
\int_{a}^{b} F^{\prime}(x) d x=F(b)-F(a)
$$

deja de tener sentido. Volterra destruyó cualquiera esperanza de establecer un Teorema Fundamental del Cálculo simple en la teoría de integración de Riemann.

A pesar del ejemplo presentado por Volterra, en 1875 Darboux tuvo éxito en determinar la propiedad de la derivada $F^{\prime}$ para que el teorema fundamental del cálculo sea verdadero en la teoría de integración de Riemann, resultado que enuncia en el siguiente teorema y es una generalización del Teorema 2, (Botsko, 1991).

Teorema 4: Sea $F:[a, b] \rightarrow \mathbb{R}$ una función diferenciable en $[a, b]$. Si $f=F^{\prime}$ es Riemann integrable en $[a, b]$, (y, por ende, acotada), entonces

$$
\int_{a}^{b} f(x) d x=\int_{a}^{b} F^{\prime}(x) d x=F(b)-F(a)
$$

\section{Demostración:}

Sea $p=\left\{x_{0}, \ldots, x_{n}\right\}$ una partición de $[a, b]$, donde

$$
a=x_{0}<x_{1}<\ldots<x_{n}=b
$$

Entonces

$$
\begin{aligned}
F(b)-F(a) & =F\left(x_{n}\right)-F\left(x_{0}\right) \\
= & \left(F\left(x_{1}\right)-F\left(x_{0}\right)\right)+\left(F\left(x_{2}\right)-F\left(x_{1}\right)\right)+\ldots+\left(F\left(x_{n}\right)-F\left(x_{n-1}\right)\right) \\
= & \sum_{i=1}^{n}\left(F\left(x_{i}\right)-F\left(x_{i-1}\right)\right)
\end{aligned}
$$

Como $F$ es diferenciable en $\left[x_{i-1}, x_{i}\right]$, por el Teorema del Valor Medio de Lagrange, existe un $c_{i} \in\left(x_{i-1}, x_{i}\right)$ tal que

$$
F\left(x_{i}\right)-F\left(x_{i-1}\right)=f\left(c_{i}\right)\left(x_{i}-x_{i-1}\right)
$$


Por consiguiente

$$
F(b)-F(a)=\sum_{i=1}^{n} f\left(c_{i}\right)\left(x_{i}-x_{i-1}\right)
$$

Note que

$$
m_{i}=\inf \left\{f(x): x \in\left[x_{i-1}, x_{i}\right]\right\} \leq f\left(c_{i}\right) \leq \sup \left\{f(x): x \in\left[x_{i-1}, x_{i}\right]\right\}=M_{i}
$$

para todo $i=1,2, \ldots, n$. Por lo tanto

$$
L(f, P):=\sum_{i=1}^{n} m_{i}\left(x_{i}-x_{i-1}\right) \leq \sum_{i=1}^{n} f\left(c_{i}\right)\left(x_{i}-x_{i-1}\right) \leq \sum_{i=1}^{n} M_{i}\left(x_{i}-x_{i-1}\right)=: U(f, P)
$$

de donde

$$
L(f, P) \leq F(b)-F(a) \leq U(f, P)
$$

para toda partición $P$ de $[a, b]$. Como

$$
\begin{aligned}
& \int_{\underline{a}}^{b} f(x) d x:=\sup \{L(f, P): P \text { es una partición de }[a, b]\} \\
& \int_{a}^{\bar{b}} f(x) d x:=\inf \{U(f, P): P \text { es una partición de }[a, b]\}
\end{aligned}
$$

se tiene que

$$
\int_{\underline{a}}^{b} f(x) d x \leq F(b)-F(a) \leq \int_{a}^{\bar{b}} f(x) d x
$$

Por hipótesis $f=F^{\prime}$ es Riemann integrable en $[a, b]$, o sea que

$$
\int_{\underline{a}}^{b} f(x) d x=\int_{a}^{\bar{b}} f(x) d x=\int_{a}^{b} f(x) d x
$$

Por consiguiente,

$$
\int_{a}^{b} f(x) d x=\int_{a}^{b} F^{\prime}(x) d x=F(b)-F(a)
$$

Una modesta generalización del Teorema 4 es la siguiente:

Teorema 5: Sean $f, F:[a, b] \rightarrow \mathbb{R}$ funciones y $E$ un subconjunto finito de $[a, b]$. Si

i) $\quad F$ es continua en $[a, b]$

ii) $\quad F^{\prime}(x)=f(x)$, para todo $x \in[a, b]-E$

iii) $\quad f$ es Riemann integrable en $[a, b]$

\section{Entonces}




$$
\int_{a}^{b} f(x) d x=\int_{a}^{b} F^{\prime}(x) d x=F(b)-F(a)
$$

\section{Demostración:}

Sea $P_{E}=\left\{x_{0}, x_{1}, \ldots, x_{n}\right\}$ una partición de $[a, b]$ tal que $E \subset P y a=x_{0}<x_{1}<\ldots<x_{n}=b$ Entonces

$$
\begin{aligned}
F(b)-F(a) & =F\left(x_{n}\right)-F\left(x_{0}\right) \\
= & \sum_{i=1}^{n}\left(F\left(x_{i}\right)-F\left(x_{i-1}\right)\right)
\end{aligned}
$$

Como $F$ es continua en $\left[x_{i-1}, x_{i}\right]$ y diferenciable en $\left(x_{i-1}, x_{i}\right)$, por el Teorema del Valor Medio de Lagrange, existe un $c_{i} \in\left(x_{i-1}, x_{i}\right)$ tal que

$$
F\left(x_{i}\right)-F\left(x_{i-1}\right)=f\left(c_{i}\right)\left(x_{i}-x_{i-1}\right)
$$

Por consiguiente,

$$
F(b)-F(a)=\sum_{i=1}^{n} f\left(c_{i}\right)\left(x_{i}-x_{i-1}\right)
$$

Como $f$ es acotada en $[a, b]$,

$$
m_{i}=\inf \left\{f(x): x \in\left[x_{i-1}, x_{i}\right]\right\} \leq f\left(c_{i}\right) \leq \sup \left\{f(x): x \in\left[x_{i-1}, x_{i}\right]\right\}=M_{i}
$$

para todo $i=1,2, \ldots, n$. Por lo tanto,

$$
L\left(f, P_{E}\right)=\sum_{i=1}^{n} m_{i}\left(x_{i}-x_{i-1}\right) \leq \sum_{i=1}^{n} f\left(c_{i}\right)\left(x_{i}-x_{i-1}\right) \leq \sum_{i=1}^{n} M_{i}\left(x_{i}-x_{i-1}\right)=U\left(f, P_{E}\right)
$$

de donde

$$
L\left(f, P_{E}\right) \leq f(b)-f(a) \leq U\left(f, P_{E}\right)
$$

para toda partición $P_{E}$ de $[a, b]$ tal que $E \subset P_{E}$.

Como

$$
\begin{aligned}
\int_{\underline{a}}^{b} f(x) d x: & =\sup \{L(f, P) / P \text { es una partición de }[a, b]\} \\
& =\sup \left\{L\left(f, P_{E}\right) / P_{E} \text { es una partición de }[a, b] \text { y } E \subset P\right\} \\
\int_{a}^{b} f(x) d x & =\inf \{U(f, P) / P \text { es una partición de }[a, b]\} \\
& =\inf \left\{U\left(f, P_{E}\right) / P_{E} \text { es una partición de }[a, b] \text { y } E \subset P\right\}
\end{aligned}
$$


se tiene que

$$
\int_{\underline{a}}^{b} f(x) d x \leq F(b)-F(a) \leq \int_{a}^{\bar{b}} f(x) d x
$$

Luego, como $f$ es Riemann integrable en $[a, b]$, se tiene que $F^{\prime}$ es Riemann integrable en $[a, b] \mathrm{y}$

$$
\int_{a}^{b} f(x) d x=\int_{a}^{b} F^{\prime}(x) d x=F(b)-F(a)
$$

En el Teorema 4 presentado por Darboux, se supone que la función $f=F^{\prime}$ es Riemann integrable en $[a, b]$. Pero ¿ ¿bajo qué condiciones la derivada $F^{\prime}$ de $F$ es Riemann integrable (sin hacer uso de la teoría de medida)?. En lo que sigue se dará respuesta a esta pregunta (Brand, 1995; Propp, 2013).

Teorema 6: Sea $\phi:[a, b] \rightarrow \mathbb{R}$ una función Riemann integrable en $[a, b]$ tal que

$$
m \leq \phi(x) \leq M
$$

para todo $x \in[a, b]$. Considere la función $\rho:[a, b] \rightarrow \mathbb{R}$ definida por

$$
\rho(x)=\int_{a}^{x} \phi(t) d t
$$

Si $\rho$ es diferenciable en $x \in[a, b]$, entonces

$$
m \leq \rho^{\prime}(x) \leq M
$$

\section{Demostración:}

Considere la función $K:[a, b] \rightarrow \mathbb{R}$ definida por

$$
K(x)=\rho(x)-m(x-a)
$$

Entonces $K$ es diferenciable en $[a, b]$ y

$$
K^{\prime}(x)=\rho^{\prime}(x)-m
$$

para todo $x \in[a, b]$. Además,

$$
\begin{aligned}
K(x) & =\int_{a}^{x} \phi(t) d t-m(x-a) \\
& =\int_{a}^{x} \phi(t) d t-\int_{a}^{x} m d t
\end{aligned}
$$


62. Visión Antataura, Vol.4, No.1, Junio - Noviembre 2020

$$
=\int_{a}^{x}(\phi(t)-m) d t
$$

Como $\phi(t) \geq m$ para todo $t \in[a, b], K$ es una función creciente en $[a, b]$. Por lo tanto, $K^{\prime}(x) \geq 0$ para $x \in[a, b]$. Así pues,

$$
\rho^{\prime}(x) \geq m, \text { para todo } x \in[a, b]
$$

Por otro lado, considere la función $L:[a, b] \rightarrow \mathbb{R}$ definida por

$$
L(x)=M(x-a)-\rho(x)
$$

Entonces $L^{\prime}$ es diferenciable en $[a, b]$ y

$$
L^{\prime}(x)=M-\rho^{\prime}(x)
$$

para todo $x \in[a, b]$. Además

$$
\begin{aligned}
L(x) & =M(x-a)-\rho(x) \\
& =\int_{a}^{x} M d x-\int_{a}^{x} \phi(t) d t \\
& =\int_{a}^{x}(M-\phi(t)) d t
\end{aligned}
$$

Como $\phi(t) \leq M$ para todo $t \in[a, b], L$ es una función creciente en $[a, b]$. Por lo tanto $L^{\prime}(x)>0$ para todo $x \in A$. Así pues

$$
\rho^{\prime}(x) \leq M, \text { para todo } x \in[a, b]
$$

De todo lo anterior se tiene que

$$
m \leq \rho^{\prime}(x) \leq M, \text { para todo } x \in[a, b]
$$

Teorema 7: Sea $\phi:[a, b] \rightarrow \mathbb{R}$ una función integrable en $[a, b]$ y $\rho:[a, b] \rightarrow \mathbb{R}$ la función definida por

$$
\rho(x)=\int_{a}^{x} \phi(t) d t
$$

Si $\rho$ es diferenciable en $[a, b]$, entonces $\rho^{\prime}$ es integrable en $[a, b]$.

\section{Demostración:}

Sea $P=\left\{x_{0}, x_{1}, \ldots, x_{n}\right\}$ una partición de $[a, b]$, donde

$$
a=x_{0}<x_{1}<\ldots<x_{n}=b
$$


Como

$$
m_{i}:=\inf \left(\phi\left[x_{i-1}, x_{i}\right]\right) \leq \phi(t) \leq \sup \left\{\phi\left[x_{i-1}, x_{i}\right]\right\}=: M_{i}
$$

Para todo $t \in\left[x_{i-1}, x_{i}\right]$, por el Teorema 6 se tiene que

$$
m_{i} \leq \rho^{\prime}(t) \leq M_{i}
$$

para todo $i=1,2, \ldots, n$. Por lo tanto,

$$
L(\phi, P) \leq L\left(\rho^{\prime}, P\right)<U\left(\rho^{\prime}, P\right) \leq U(\phi, P)
$$

y

$$
\int_{\underline{a}}^{b} \phi(x) d x \leq \int_{\underline{a}}^{b} \rho^{\prime}(x) d x \leq \int_{a}^{\bar{b}} \rho^{\prime}(x) d x \leq \int_{a}^{\bar{b}} \phi(x) d x
$$

Como $\phi$ es integrable en $[a, b]$, se tiene que

$$
\int_{\underline{a}}^{b} \phi(x) d x=\int_{a}^{\bar{b}} \phi(x) d x=\int_{a}^{b} \phi(x) d x
$$

Por consiguiente,

$$
\int_{\underline{a}}^{b} \rho^{\prime}(x) d x=\int_{a}^{\bar{b}} \rho^{\prime}(x) d x
$$

y $\rho^{\prime}$ es integrable en $[a, b]$.

Teorema 8: Sea $F:[a, b] \rightarrow \mathbb{R}$ una función diferenciable en $[a, b]$. La derivada $F^{\prime}$ es integrable en $[a, b]$ sí y sólo si, existe una función integrable $\phi:[a, b] \rightarrow \mathbb{R}$ tal que

$$
F(x)=F(a)+\int_{a}^{x} \phi(t) d t
$$

para todo $x \in[a, b]$.

\section{Demostración:}

Si $F^{\prime}$ es integrable en $[a, b]$; entonces por el Teorema 4,

$$
\int_{a}^{x} F^{\prime}(t) d t=F(x)-F(a)
$$

Luego, 
64. Visión Antataura, Vol.4, No.1, Junio - Noviembre 2020

$$
F(x)=F(a)+\int_{a}^{x} F^{\prime}(t) d t=F(a)+\int_{a}^{x} \phi(t) d t
$$

donde $\phi:=F^{\prime}$ es una función integrable en $[a, b]$.

Recíprocamente, supongamos que existe una función integrable $\phi:[a, b] \rightarrow \mathbb{R}$ tal que

$$
F(x)=F(a)+\int_{a}^{x} \phi(t) d t
$$

para todo $x \in[a, b]$. Sea $\varphi:[a, b] \rightarrow \mathbb{R}$ la función definida por

$$
\rho(x)=\int_{a}^{x} \phi(t) d t
$$

Luego

$$
\rho(x)=F(x)-F(a)
$$

para todo $x \in[a, b]$. Por lo tanto, $\rho$ es diferenciable en $[a, b]$ y $\rho^{\prime}=F^{\prime}$. Así, por el Teorema $7, F^{\prime}=\rho^{\prime}$ es integrable en $[a, b]$.

Conclusión: Sea $F:[a, b] \rightarrow \mathbb{R}$ una función diferenciable en $[a, b]$. Si existe una función integrable $\phi:[a, b] \rightarrow \mathbb{R}$ tal que

$$
F(x)=F(a)+\int_{a}^{x} \phi(t) d t
$$

para todo $x \in[a, b]$, entonces por el Teorema 8 , la función $\rho:[a, b] \rightarrow \mathbb{R}$ definida por

$$
\rho(x)=\int_{a}^{x} \phi(t) d t
$$

es diferenciable en $[a, b]$ y $\rho^{\prime}=F^{\prime}$. Luego, por el Teorema $7, F^{\prime}=\rho^{\prime}$ es integrable en $[a, b]$. Por consiguiente, por el Teorema 4,

$$
\int_{a}^{x} F^{\prime}(t) d t=F(x)-F(a)=\int_{a}^{x} \phi(t) d t
$$

Sin embargo, esto no implica que $F^{\prime}=\phi$, como lo muestra el siguiente ejemplo

Ejemplo 4: Sean $F, \phi:[-1,1] \rightarrow \mathbb{R}$ las funciones definidas por

$$
F(x)=\left\{\begin{array}{ll}
0,-1 \leq x<0 \\
x^{2}, & 0 \leq x \leq 1
\end{array} \quad \phi(x)= \begin{cases}0, & ,-1 \leq x<0 \\
2, & x=0 \\
2 x & , 0<x \leq 1\end{cases}\right.
$$


Entonces $F$ es diferenciable en $[-1,1]$ y

$$
F^{\prime}(x)= \begin{cases}0 & ,-1 \leq x<0 \\ 2 x & , 0 \leq x \leq 1\end{cases}
$$

Además,

$$
\int_{-1}^{x} F^{\prime}(t) d t=\int_{-1}^{x} \phi(t) d t
$$

para todo $x \in[-1,1]$. Sin embargo $F^{\prime} \neq \phi$ en $[-1,1]$

\section{Referencias bibliográficas}

Barthle, D.R. and Sherbert, R. G. (2011). Introduction to Real Analysis. U.S.A.: John Wiley \& Sons.

Botsko, M.W. (1991). A fundamental Theorem of Calculus that Applies to all Riemann Integrable Functions. Mathematics Magazine 64 (5), 347-348.

Brand, L. (1995). The Fundamental Theorem of Calculus. The American Mathematical Monthly 102 (5), 440-441.

Bressoud, D.M. (2011). Historical Reflections on Teaching the Fundamental Theorem of Integral Calculus. The American Mathematical Monthly 118 (2), 99-115.

Dunham, W. (2005). The Calculus Gallery: Masterpieces from Newton to Lebesque. U.S.A: Princeton University Press.

Edwards, C. H. Jr. (2013). The Historical Development of the Calculus. U.S.A.: Springer

Propp, J. (2013). Real Analysis in Reverse. The American Mathematical Monthly 120 (5), $392-408$. 